\title{
Polysèmes
}

Revue d'études intertextuelles et intermédiales

\section{In Arcadia, de Ben Okri : du topos littéraire à l'énigme du tableau}

Isabelle Gadoin

\section{(2) OpenEdition}

1 Journals

Édition électronique

URL : http://journals.openedition.org/polysemes/2572

DOI : 10.4000/polysemes. 2572

ISSN : 2496-4212

Éditeur

SAIT

\section{Référence électronique}

Isabelle Gadoin, «In Arcadia, de Ben Okri : du topos littéraire à l'énigme du tableau », Polysèmes [En ligne], 19 | 2018, mis en ligne le 30 juin 2018, consulté le 10 décembre 2020. URL : http:// journals.openedition.org/polysemes/2572; DOI : https://doi.org/10.4000/polysemes.2572

Ce document a été généré automatiquement le 10 décembre 2020.

Polysèmes 


\title{
In Arcadia, de Ben Okri : du topos littéraire à l'énigme du tableau
}

\author{
Isabelle Gadoin
}

1 In Arcadia, publié en 2002, est un récit tout à la fois atypique et révélateur de la production de Ben Okri; atypique en ce qu'il s'écarte du contexte nigérian, et plus largement post-colonial du reste de ses romans, nouvelles et poèmes; et cependant révélateur car il exploite la même veine métaphorique et allégorique que des fables telles que Astonishing the Gods (1995), qui contait sur un mode des plus cryptiques, celui de la sentence ou de la maxime - souvent énoncée comme paradoxe - la découverte d'une contrée onirique où se matérialisent les pensées et où s'incarne une sagesse utopique, qui n'est autre qu'un parfait humanisme. Mais c'est sans doute par là que resurgit la dimension post-coloniale et critique du travail de Okri : dans la figure récurrente du voyage initiatique, de l'exil d'un pays, et de la rencontre d'une philosophie, ou mieux d'une éthique, qui soit enracinée dans un lieu. Or In Arcadia prolonge cette réflexion sur le sens du lieu, et le lieu du sens.

2 Le récit suit en effet le voyage de sept personnages partis de Londres pour se rendre en Grèce, dans la région nommée "Arcadie », mais les abandonne en cours de route, au musée du Louvre, devant le tableau de Poussin intitulé Les Bergers d'Arcadie, lieu d'une révélation épiphanique. Le lecteur saisit progressivement et presque à mots couverts que Lao, le narrateur qui ouvre le récit, est noir, et que le voyage qui le confronte aux Londoniens est aussi une réflexion sur la race et la fraternité. Mais mon interrogation s'éloignera de ce contexte post-colonial - sans doute pour mieux y revenir, car je voudrais me concentrer sur la figure de l'Arcadie, qui ne peut par définition s'écarter longtemps du concept de lieu idéal.

3 Mon approche cependant sera plus théorique que politique, car centrée sur la question du rapport texte-image, dont Ben Okri propose une exploration tout à fait originale dans ce récit qu'on ose à peine qualifier de roman tant la dimension métaphorique y est insistante. Comme le pose explicitement le titre, le récit s'inscrit dans la longue tradition mythique, littéraire puis picturale de l'Arcadie - ce que j'appellerai, dans un premier moment de cette étude, le «topos» arcadien. Rapidement toutefois Okri déplace, 
dénonce, ou désactive le lieu commun pour le transformer en séries de métaphores fluides, labiles. Chacun des personnages tour à tour tente de dire à travers la figure de l'Arcadie ce que signifie ou incarne pour lui le lieu rêvé. Ce récit touche donc lui aussi à la fable, le voyage dans l'espace devenant peu à peu exploration personnelle, cheminement intérieur. L'Arcadie par essence inaccessible devient alors énigme, l'énigme de ce qu'opère pour chacun le rêve d'un ailleurs. Et c'est sans doute là la réussite d'Okri dans ce récit: utiliser l'Arcadie non plus comme lieu commun ou comme figure, mais comme l'énigme qui finit par rythmer la narration et ponctuer la lecture. Okri parviendrait donc ici à une mise en œuvre du pictural dans le texte qui ne soit pas du ressort du mimétique, ni même plus largement de l'iconique, car le tableau de Poussin qui sous-tend toute la quête n'est en fait jamais saisi ni "cadré » par une écriture qui serait de l'ordre du descriptif, et il se dérobe sans cesse à la lecture, au grand dam du lecteur friand d'images à voir sous les images à lire... Et si l'image vient ici dynamiser le texte, c'est plutôt par son potentiel narratif, sa capacité à lancer le récit et à le maintenir toujours ouvert, interprétable à l'envi, jamais clos.

\section{Le « topos»}

Les dictionnaires et les encyclopédies nous enseignent que l'Arcadie fut un pays bien réel, dressant au centre du Péloponnèse « ses austères montagnes de marbre et de schiste $»^{1}$. À cet isolement géographique, la contrée doit d'être devenue synonyme de retraite heureuse et protégée, dans la tradition. Pour le reste, l'article de l'Encyclopédie brosse un tableau plutôt lugubre de la situation arcadienne, territoire dépeuplé, à l'agriculture déclinante, aux villages décimés. L'historien d'art Erwin Panofsky, étudiant la tradition arcadienne dans son article "Et in Arcadia Ego: Poussin et la tradition élégiaque ", oppose ce qu'il nomme à la suite d'historiens de l'Antiquité le «primitivisme dur » et le "primitivisme doux». L'Arcadie réelle, résume-t-il, était une terre "pauvre, aride, rocailleuse, peu avenante, dénuée de tous les agréments de la vie et fournissant à peine de quoi nourrir quelques chèvres efflanquées ». Ses habitants étaient connus pour leurs talents musicaux, «l'austérité de leurs mœurs, leur hospitalité rustique » (Panofsky 282) " mais aussi leur noire ignorance, leur bas niveau de vie n'étaient pas moins notoires » (281). C'est au fil du traitement du thème par les poètes, Théocrite dans ses Idylles tout d'abord, puis Virgile dans ses Bucoliques, que prit forme l'Arcadie telle que nous la rêvons aujourd'hui, « royaume fictif de parfaite félicité » (284), Arcadie d'utopie. Dans ce cadre naturel parfait, cependant, Virgile introduit une dissonance avec l'épisode de la mort de Daphnis châtié par Aphrodite pour avoir défié la toute-puissance de l'amour, et à qui ses amis se destinent à ériger un monument funéraire. Voici inventé le thème élégiaque de la «tombe en Arcadie», qui signale la présence incontournable de la mort, menaçant chacun, même au cœur du paysage le plus idyllique.

Virgile ne semble donc adoucir les traits du paysage, en faire un havre de paix, que pour mieux renforcer le sens d'un présent contesté. Il invente, selon Panofsky, cette " alliance vespérale de mélancolie et de sérénité » (284), cette prescience ambiguë d'un bonheur humain condamné à n'être qu'éphémère. De la tradition littéraire qu'initie Virgile cependant, seule semble avoir été retenue la capacité des bergers d'Arcadie à oublier leur malheur (c'est-à-dire tout simplement leur statut de mortels) en rivalisant de chants dans un décor champêtre idéal. Et l'image sombre s'allégea en visions de l'Arcadie comme 
domaine enchanté où Pan fait danser au son de sa flûte des cortèges enjoués de nymphes, naïades, dryades et autres divinités des bois.

6 C'est cette dernière Arcadie, utopie qui lisse les aspérités et les menaces qui troublaient encore le royaume bucolique de Virgile, qui lance le récit de Ben Okri : sept personnages en quête d'ailleurs sont payés pour aller réaliser un documentaire télévisé en Arcadie, et même s'il s'agit bien là de la dure région de Grèce « démasquée » par Panofsky, c'est sous la forme du cliché arcadien déjà constitué que l'endroit leur est présenté. Ce cliché est formé de toutes les stratifications et les condensations du thème, rejoignant paradoxalement, bien au-delà de la mythologie de Théocrite et de Virgile, le thème et l'imagerie biblique : « How did it all start, that's what I am asking myself? As far as I can make out it began with someone having the crankiest notion, in our cynical times, that we should make a film about a journey to Arcadia, a place of rural tranquillity, a sort of Garden of Eden, our lost universal childhood » $(\text { Okri 7 })^{2}$.

7 Le niveau de surgissement de l'Arcadie dans le texte est donc celui du topos, du lieu commun, au sens à la fois littéral (la région de Grèce, et donc le lieu dit en Grec) et figuré (le lieu d'un savoir commun cristallisé à travers les âges). Le topos n'a pas à être défini, ou bien alors seulement de manière minimale et approximative, comme « $a$ sort of Garden of Eden » (c'est moi qui souligne). Peut-être même ne peut-il pas être défini : il est transmis par la rumeur, le on-dit. Le topos est de l'ordre du « donné », d'un « toujours-déjà là » qui s'inscrit comme l'un des multiples maillons d'une longue filiation littéraire, qui peut échapper à l'individu, mais appartient toujours à sa culture collective : "It was an intriguing project, a place we'd never heard of, a place called Arcadia, a place that's supposed to be loaded with classical allusion» $(5$; je souligne). Le topos «arcadien » est donc posé, et imposé à un narrateur bougon qui ne le reçoit qu'à contrecœur et ne l'accepte que comme fuite, comme échappée hors d'un quotidien morose, ainsi qu'il l'explique en prélude, à la toute première page du récit : «We didn't give a damn about [Arcadia]. All we wanted was to work again, to be on the road again, away from all the problems, all the failures, all the messed-up relationships " (5). Le topos, lieu commun, peut donc bien véhiculer toute une série d'images, le narrateur n'en a cure, ne veut pas en entendre parler, et ses imprécations font systématiquement retomber l'image idéale au niveau le plus trivial :

Why anyone would want to make a barmy film like that is beyond me. Who cares if we've lost our childhood, or if we've lost our way? Who gives a chicken's fart about the Garden of Eden and rural tranquillity and improbable things like that? No one thinks about that stuff any more. No one believes in it. All we care about is the next paypacket, the next meal, the next gratification, the next party, the next football match, the next sensation. (8)

Rarement on aura vu narrateur aussi mal disposé, aussi gratuitement fielleux. Lui-même confie d'ailleurs se délecter de son cynisme universel - et il faut bien avouer que ce n'est pas non plus sans une certaine jouissance (voyeuriste ? sadique ?) que le lecteur s'apprête à suivre malgré lui ce guide fermé à toute découverte, et dont les torrents de bile vont, ironiquement, à contre-courant de son rôle imposé d'éclaireur :

My heart is ash. My feelings are frozen. My eyes are dead. My thoughts are cold. Nothing stirs in me. Nothing surprises me. I expect the worst. Human beings stink, that's a fact. And so when some idealist comes along with some sentimental notion about finding ourselves again and rural tranquillity I sort of get murderous. They make me edgy; they get on my nerves. (9) 
Le lecteur en vient à soupçonner que ces trésors d'acrimonie ne révèlent en fait que la violence de la dénégation. Car clamer comme le fait le narrateur que sa vie et le monde moderne tout entier sont des « enfers» (« the daily round in this inferno that we call the modern world», 5), n'est-ce pas poser par l'absurde la possibilité d'un monde meilleur, d'une Arcadie salvatrice ? La phrase d'ouverture de l'incipit a d'ailleurs donné la clé de ce traitement volontairement à contre-ton de ce qu'impliquait le titre de l'ouvrage, In Arcadia : " In our different ways, we were all on the verge of nervous breakdown when the message got through » (5). Il faut évidemment que le récit débute dans ce total désespoir pour qu'une Arcadie puisse être découverte. Que les personnages soient sauvés. Et qu'ait lieu l'épiphanie finale. L'amertume du narrateur se comprend dès lors comme l'exact inverse du sentiment bucolique qui persisterait à chanter l'espérance depuis le cœur de la douleur même, de la confrontation avec la mort. Surmontant le scepticisme du héros malgré lui, le voyage imposé sera peut-être le passage des affres de l'enfer moderne à l'espoir ou à la régénération, dans le lieu promis.

\section{La fable}

L'odyssée commune, ironiquement rétrécie à un voyage en Eurostar de Londres à Paris, se révèle donc rapidement être un grand voyage symbolique, sorte de nef des fous des temps modernes - la «stultifera navis » dont Michel Foucault rappelle dans son Histoire de la folie à l'âge classique qu'elle fut elle aussi un thème littéraire avant de devenir motif pictural sous la main de peintres comme Jérôme Bosch ${ }^{3}$ : «Life is clearly akin to a long spell in prison, a long illness with no remission, a nightmare, a hell-hole, a freak show, a ship of hypocrites, a house of opportunists, a landscape of fools » (9). Fous, les personnages le sont d'ailleurs tous d'une manière ou d'une autre, "shipwrecks and derelicts ", " all wretches clinging on to sanity's last nerve » (5). Chacun se débat dans ses obsessions, pour l'argent, le travail, l'autorité, la parole (logorrhée plutôt), etc., et le début du récit les présente moins comme une galerie de portraits réalistes que comme une série de types, caricatures proches des incarnations médiévales des sept péchés capitaux. La métaphore de cette nef des fous fait donc du récit un conte et une moralité, plaçant les personnages dans l'espace du sacré. Car, comme l'étrange "contre pèlerinage » qui lançait la nef des fous sur toutes les routes dans l'espoir de les voir purger leur folie et trouver un lieu sauf, les damnés de l'existence embarqués malgré eux dans le voyage de tournage du documentaire télévisé sur l'Arcadie attendent vaguement une guérison miraculeuse : «We were, in our different ways, still hoping for something to turn up and save us from the abyss. Some scrap of luck, a miracle, a piece of good fortune a new kind of bible that would straighten out the awful miserable mess of our lives » (5). C'est l'image de cette nef tentant d'échapper à la folie qui fait passer le récit du lieu commun à la métaphore. Car c'est bien du néant de l'existence qu'il est question, et de stratégies désespérées pour y échapper.

Or ces personnages sont poursuivis. Chacun d'entre eux, au fur et à mesure du voyage, se voit envoyer un "message ». Le message apparaît, brûlant sur un papier rouge sang, au creux de leur paume. En théorie, c'est l'organisateur de l'expédition, un certain Malasso au nom bien inquiétant, qui est censé envoyer aux membres du tournage les consignes nécessaires au périple. Mais ce Malasso devient vite une abstraction, un anonyme que nul ne connaît ni n'a jamais vu. Interloqués, les destinataires des messages refusent obstinément d'en révéler la teneur à leurs compagnons de voyage, et le lecteur n'aura 
jamais accès aux mots qui terrorisent. Seul est dévoilé le message adressé en premier lieu à Lao, une mise en garde qui intrigue plutôt qu'elle n'éclaire le lecteur: "Beware the inscriptions »... Peu à peu la source des messages se dématérialise, et le narrateur philosophe : «Every now and again life sends us little messages. The messages are meant for us alone. No one else can see them. No one else perceives them as messages. They may seem perfectly banal to the world, but to you, for whom they were intended, they have the force of revelation " (22). L'existence tout entière consiste donc à tenter de déchiffrer les messages que nous envoie la vie - et peut-être même l'au-delà de la vie. C'est ce que comprend Lao au seuil du périple : «I must [...] make a new covenant. I must find a way to make death not a threat, an enemy, a terror, an excuse, but a friend, an aid, a liberator. For it would seem that death is the golden key to the mystery of living, but I don't know how to use it " (32). Ce sens de l'immanence de la mort au sein de la vie, «the finality of death, the impermanence of death » (77), ne quittera aucun des personnages, jusqu'aux appels lancinants qui ponctuent le dernier livre tel un leitmotiv: "Is it death that secretly troubles us?» $(225,227,230)$. La terreur est donc toujours là, qui se double évidemment de l'angoisse du temps, perceptible dès l'instant où les voyageurs-pèlerins se rassemblent sous l'horloge de la gare de Waterloo, "under the shadow of the clock» (40) :

Every journey is a little dying. Death is the train on which we travel [...]. Death is the vehicle of the voyage, but death is never its destination [...] A life rests in death; that is not a destination. A destination is different from a destiny. (49)

12 Au milieu de cette angoisse latente, les messages secrets assénés à chacun sonnent comme des appels ou peut-être des rappels de l'au-delà, et sont reçus comme autant de coups de semonce. Le lecteur les voit, du dehors, agir individuellement sur chaque personnage comme une épiphanie, le glaçant d'effroi, mais le transformant aussi définitivement, l'humanisant peu à peu, lui donnant la capacité à fraterniser qui faisait cruellement défaut à tous au début du voyage. «I wasn't the same afterwards. I was never the same again » dit Lao (26). Les liens pourraient se créer, les personnages en arriver à communiquer, si ce n'est s'entraider. Par les révélations de feu au creux de leurs paumes se révèle leur commune humanité. Les voyageurs peu à peu parlent, se confient sur ce que serait leur propre Arcadie, se plaisent à y voyager en imagination, à la décrire à leurs compagnons. C'est Lao qui propose le jeu de la révélation, « to reveal what their private Arcadia would be » (134). Malgré toutes les confidences cependant le concept d'Arcadie reste flou, série d'esquisses ou de variations sur le thème :

Propr: "I don't understand this arcadian business" (134)

Sam: "I am sort of sympathetic to this Arcadia thing" (140)

Jute: "I think work is Arcadia" (143)

Jim: "to me the most important thing is not that which does the awakening, but the awakening itself [...]. The true Arcadian notion belongs to the future of humankind. We haven't realised it yet" (147)

C'est là que l'objet de la quête s'inverse, s'intériorise, et que le voyage réel s'efface devant la représentation personnelle de l'Arcadie: «Maybe the healing must begin within, thought Lao » (225). Le lecteur comprend peu à peu qu'il est lui-même en train de cheminer dans des lieux irréels, parmi des images, des représentations d'Arcadie, entre lesquelles s'impose irrésistiblement le tableau de Poussin intitulé Les Bergers d'Arcadie.

Dans ce tableau, trois bergers méditent et discourent sur une tombe portant pour seule inscription «Et in Arcadia ego », formule dont Panofsky analyse longuement l'ambiguïté, si ce n'est le double sens, dans l'un de ses articles. Car, démontre-t-il, ce que nous croyons 
aujourd'hui déchiffrer linéairement dans l'expression latine («Et moi aussi j’ai vécu / ou je suis en Arcadie ») est en fait un contresens sur le latin, dans lequel la coordination et ne peut porter que sur le terme qui la suit immédiatement, tandis que le verbe en ellipse dans la phrase ne peut être que la particule existentielle "esse ", nécessairement sousentendue au présent. Le sens exact de la formule est donc « Et même en Arcadie, moi je suis ", qu'on peut tout au plus extrapoler comme «je suis présent(e) ». Reste à savoir à qui ce « je » fait référence. Au berger mort ? Ou plutôt à la mort elle-même, parlant par prosopopée à travers l'inscription? C'est là le sens initial de la formule, et Panoksky rétablit certains des maillons de la tradition picturale exhibant ce sens sans ambiguïté : dans un tableau du Guerchin exécuté à Rome entre 1621 et 1623 et conservé à la galerie Corsini, l'inscription figure déjà sur un tombeau surmonté d'une tête de mort, qui fait de l'œuvre une claire mise en garde, « un memento mori médiéval sous travesti humaniste : un thème cher à la théologie morale chrétienne, transposé dans l'ambiance idéale des pastorales classiques» (Panofsky 292). Or il existe deux tableaux de Poussin intitulés Les Bergers d'Arcadie. Le premier, qui figure dans la collection du duc de Devonshire à Chatsworth House, fait encore apparaître un crâne, certes difficilement visible sur la pierre tombale, mais dont la présence, même effacée, situe encore le tableau dans cette tradition bien connue du memento mori. Le second tableau en revanche, celui du Louvre, en effaçant le crâne et les signes qui désignent trop ostensiblement la mort, désarticule la phrase « et in Arcadia ego » de tout énonciateur, et en semant le doute sur le destinateur et le destinataire du message, ouvre le sens du tableau. Tel est, suggère Panofsky, le coup de génie de Poussin: avoir extrait ce type de représentation de la tradition morale à laquelle il appartenait, proche de la vanité, pour le placer dans un contexte plus doucement élégiaque: "l'attitude des personnages n'exprime plus ni surprise ni effroi mais la méditation et le recueillement du souvenir » (Panofsky 298).

On comprend à ce long détour par l'exégèse picturale que le récit de Ben Okri ne dissémine rien d'autre que la question du tableau de Poussin et ses répercussions obsédantes : « is it death that secretly troubles us?». Tout comme le tableau nous renvoie son énigme sur l'entrelacs de la vie et de la mort, les messages envoyés aux personnages les interrogent, les interloquent à proprement parler, sur leur propre compréhension de la condition mortelle. Une correspondance subtile s'établit entre le mystère du tableau et le mystère de l'aventure, comme si l'effet de la toile percolait lentement à travers le texte.

\section{L'énigme}

Dans ses analyses approfondies du thème de "Et in Arcadia ego ", Panofsky fait, bizarrement, œuvre de philologue plus que d'historien de l'art : il se penche sur l'énigme des signifiants, la double signification de l'inscription, mais n'interroge pas, ou très peu, la structure du tableau. Or, me semble-t-il, pour revenir sur ses analyses, une différence majeure oppose les deux versions du thème arcadien données par Poussin. La première, et la moins connue, est une composition axiale: les bergers, encore en mouvement, s'arrêtent, surpris dans leur promenade par la découverte d'un tombeau, dont ils déchiffrent l'inscription «Et in Arcadia Ego », difficilement perceptible, sur le côté droit de la toile.

17 Le tableau du Louvre, ultérieur, diffère principalement de la première version par sa composition, rigoureusement centrale. L'élément de surprise est atténué car les bergers ne sont plus arrêtés dans leur course mais ont déjà mis genou à terre, et sont penchés de 
part et d'autre du tombeau, affairés à tenter de déchiffrer l'inscription, vue frontalement cette fois. Or le positionnement central de l'inscription dans cette composition lui permet, ce me semble, d'assurer un extraordinaire retournement du visuel au textuel: l'inscription, et son irrésistible appel à la lecture, fait en effet basculer le dispositif spectatoriel (dans lequel nous autres spectateurs, par notre propre positionnement, assignons à la construction perspectiviste son point central, son objet) en un dispositif d'énonciation, dans lequel à l'inverse c'est le point central, devenu point du sujet, qui interpelle un spectateur devenu objet - ou à tout le moins interlocuteur, puisque c'est le point central, au bout des doigts du berger, qui devient point d'origine du discours.

Ce que font les mots dans la peinture, c'est donc de renverser la chose vue en chose dite, mais aussi par là même la troisième personne du singulier, que nous construisions au cœur du tableau (« la tombe est au centre de la composition »; « la mort plane au cœur de la vie, cette vie fût-elle l'existence douce et sereine de l'Arcadie elle-même ») en une inquiétante première personne du singulier, qui nous parle et nous "point» depuis le centre du tableau, d'où les mots, véritable punctum, viennent nous percer d'une révélation: "moi la mort, je suis présente même en Arcadie». Le simple "il y a ", l'exclamation de la découverte étonnée qui pouvait être celle des bergers dans le premier tableau de Poussin, se renverse donc ici en un " ego ", le «je » d'une peinture devenue parlante. Le mot est donc le lieu où la peinture s'inverse, ou peut-être s'approfondit, en langage, et où la représentation devient réflexion sur la représentation.

Cependant je nuancerais légèrement l'analyse de W.J.T. Mitchell qui dans son ouvrage Picture Theory classe le tableau de Poussin parmi ce qu'il nomme "Metapictures »: "a representation of representation ", "a picture of textuality and reading» (Mitchell 76). Mitchell distingue en effet deux niveaux dans cette confrontation : le drame des bergers, et les interrogations du spectateur - mais il ne va pas jusqu'à préciser que ce qui fait la force de l'œuvre, c'est que chacun de ces deux niveaux peut à tout instant s'inverser en son autre, comme en miroir : le spectateur lisant l'inscription qui laisse les bergers muets de surprise, et la toile s'adressant en retour à qui la regarde. Indéfiniment.

En effet, cette interprétation ( $M$ Moi, la mort») n'est que l'un des sens possibles de l'inscription. Car cet " ego » qui y parle à la première personne est un pronom dont l'antécédent reste obstinément vide, vide comme ce tombeau du tableau où repose un corps dont l'identité n'est pas devinable. Dans les Bucoliques de Virgile, c'est Daphnis dont on célébrait la mort. Mais ici? Berger? Noble ? Qui est ce « moi », énonciation désancrée de toute origine? Est-ce nécessairement la mort? Ou encore une projection de nousmêmes qui parlerions à la mort; ou bien qui parlerions de notre mort; ou enfin qui parlerions aux vivants par delà notre mort ? Entre ce « je » (« ego ») inattribuable, le « il » d'un récit possible, et le "nous» qui place spectateurs et lecteurs dans la position d'herméneutes impuissants, le dialogue est donc infini. C'est là la force insondable de l'œuvre : la capacité du dispositif mis en place par le tableau à convoquer le spectateur et à le mettre en dialogue avec l'œuvre, c'est-à-dire à créer ce que Louis Marin appelle « la fiction d'une voix » (Marin 1995, 115). (Et il faudrait ici ouvrir tout un développement sur l'incapacité du tableau à poser avec certitude ce qui correspondrait aux pronoms personnels: évidemment, ce qui échappe au pictural, c'est le système des voix. $\mathrm{Ne}$ confondons pas : la peinture n'est jamais un dispositif d'énonciation, tout au plus peutelle le suggérer ; mais c'est précisément à cette suggestion que Poussin réussit à donner, dans le tableau du Louvre, la force d'une fascination : l'illusion, toujours poursuivie, d'une " peinture parlante». 
21 Il est d'ailleurs révélateur que la toile de Poussin, initialement intitulée Les Bergers d'Arcadie, soit passée à la postérité sous le titre Et in Arcadia Ego, par un processus qui transforme l'inscription incluse dans l'œuvre en titre de l'œuvre. L'inscription n'est pas inhabituelle chez Poussin, et ses deux autoportraits par exemple comportent de pompeuses inscriptions latines - en lettres majuscules bien sûr! L'autoportrait dit «Pointel», du nom de son collectionneur, est surmonté de la phrase « Nicola Poussin Andelyensis Academicus Romanus Primus / Pictor Ordinarius Justi Regis Galliae, Anno Domino 1649 ». L'autoportrait dit «Chantelou », du nom de son ami et mécène, inscrit en lettres d'or sur une toile vierge, juste derrière le peintre: «Effigies Nicola Poussini Andelyensis Pictoris, Anno Aetatis, Romae Anno Jubili $1650 »$. La fonction de ces inscriptions, des plus traditionnelles, est ici d'attribution, de datation, d'authentification, voire de glorification... Mais l'inscription à laquelle sont confrontés les bergers d'Arcadie agit exactement à l'inverse. Elle fait porter le doute sur des identités, des attributions possibles du discours. Elle crée le mystère du tableau, un mystère qui finit par recouvrir les certitudes mêmes de l'œuvre, c'est-à-dire son titre premier. Plus que comme un titre, elle agit donc comme une légende au sens strict du latin legendum, une indication sur la manière de lire l'œuvre. Toute la portée du tableau vient donc de l'énigme qu'il crée.

Or c'est exactement ce que Ben Okri réussit à mettre en œuvre à l'intérieur de son étrange récit. Tout comme Poussin, il fait fonctionner «la fiction d'une voix " en l'absence de toute « assignation référentielle » (Marin 1995, 115). Une voix qui parle d'on ne sait où, pour on ne sait qui : les messages inquiétants reçus par les personnages restent muets pour nous, sans contenu, puisque la teneur n'en est jamais dévoilée, mais ce faisant ils se réduisent à une fonction d'interpellation, amenant chacun des voyageurs à s'interroger profondément sur ce que signifie pour lui la notion d'Arcadie, sur le sens individuel que chacun prête au lieu rêvé, au lieu-refuge où puisse s'ignorer un temps l'immanence de la mort. Le récit dissémine donc autant d'appels de la peinture à l'interprétation (" Arcadie - votre Arcadie, quelle Arcadie?»), sans jamais fixer un sens à cette quête individuelle. C'est la fonction didactique que Mitchell reconnaît aux « metapictures »: « the continuing function of the metapicture as teaching aid » (Mitchell 77). Et c'est en second lieu ce que Ben Okri dérive du tableau : la capacité de l'énigme à irradier dans tout le récit. Celui-ci reste un récit sans réponse, n'exploitant quasiment que le mode interrogatif (dont on pourrait aisément donner une analyse stylistique, voire narratologique, dans ces chapitres entiers intitulés "Intuitions in the Garden » ou "Intuitions in the Dark », qui n'avancent que de tâtonnement en tâtonnement). Comme le tableau de Poussin, In Arcadia repose sur « a process of encounter, apprehension, puzzlement and discussion" (Mitchell 77). Enfin, le troisième type d'utilisation de l'inspiration picturale découle des deux premiers. En adoptant un système d'énonciation sans origine, et le sens de l'énigme ontologique proposée par le tableau (la vie, la mort, et leur croisement perpétuel), Okri exploite la possibilité de «mise en histoire » (Marin 1995, 115), la capacité du tableau à suggérer un récit.

Cette énigme « arcadienne » trouve son dénouement dans le moment tant attendu par le lecteur qu'est la confrontation des voyageurs avec la toile de Poussin, lorsqu'ils atteignent enfin Paris et se rendent au musée du Louvre. Or, magistrale déception! Cette confrontation est pour le lecteur à la limite de la non-rencontre. On attendait une ekphrasis, une description d'autant plus travaillée qu'elle avait été longtemps retardée dans le récit, arrivant une vingtaine de pages seulement avant sa clôture; et l'on 
n'obtient qu'une parodie d'ekphrasis, soigneusement plaquée au degré zéro de la description :

The painting appears simple. Three shepherds and a shepherdess in front of a tomb. The tomb is in a rocky landscape with a few trees. The four figures are reading the inscription on the tomb, and they appear to be trying to decipher it. The shepherdess stands slightly aloof from their puzzlement.

It is a beautiful painting, but the beauty does not reside in the landscape, which is rocky and mostly bare. The beauty is in the structure, the colour, the harmony of the lines of force in the painting, and in its mood.

At the centre of the painting is the tomb. And at the centre of the tomb is the inscription: Et in Arcadia Ego. Those four words are among the most debated in the history of art, the most enigmatic, puzzling, mysterious, and endless. (204)

De deux choses l'une: soit Okri s'est plu à ménager le suspens de la rencontre pour finalement faire montre de toute l'étendue de son inaptitude à la description " picturale », mais c'est un peu douteux étant donné le choix même de son sujet ; soit son intérêt réside tout simplement ailleurs. De fait, dans tout le récit, il court-circuite soigneusement la dimension picturale de l'œuvre, qu'il résume avec la naïveté du manuel pour Grands Débutants en «structure, colour, and harmony of the lines of force ». Dans tout son texte, aucun «marqueur du pictural» (Louvel 33), aucune allusion un tant soit peu visuelle.

Le moment de la confrontation avec le tableau de Poussin est donné comme épiphanique : « it is impossible for an intelligent human being to see this painting, to think about it, and to live the same way they lived before " (205). Jamais pourtant ne sera dévoilée la signification que prend cette épiphanie pour chacun des personnages. L'Arcadie reste abstraction, concept, rêve personnel, et Okri ne semble s'intéresser ni à la description du tableau, ni même - ce qui est plus original - à son interprétation, qu'il couche noir sur blanc, sous forme de questions et de suppositions, immédiatement après sa brève " antiekphrasis». De fait, les interprétations du tableau sont systématiquement exposées, démultipliées dans le texte, ce qui donne au lecteur le sentiment désappointant et ambigu que le texte est à la fois codé de bout en bout par la présence de l'œuvre sous l'œuvre, et en même temps toujours livré « clefs en mains » dans de grands passages plus pontifiants encore que didactiques, et en tous cas plus universitaires que romanesques... Ainsi lorsque chaque personnage se complaît à définir ce que serait son Arcadie, à la sortie du Tunnel sous la Manche, moment du retour à la lumière, le texte ne décrit rien de visuel mais se perd en longues tirades pompeuses qui n'évitent pas toujours la sentence moralisatrice.

De même, la découverte du tableau du Louvre par les voyageurs est immédiatement suivie d'une interview de Pierre Rosenberg, l'ancien directeur du musée, qui n'est pas nommé mais implicitement désigné dans le texte comme «l'homme à l'écharpe rouge ». Mais une fois encore, le texte enchaîne de pathétiques clichés sur le statut de l'artiste et une liste débridée de "mots clés" non raisonnés. Qui informent certes, si besoin était, mais laissent le lecteur sur sa faim concernant la portée réelle de la figure de l'Arcadie dans le récit :

The director of the museum wore a red scarf over his shoulder and a dark suit [...] Lao : "It is a pleasure to meet you, Director, and a pleasure to be here at the Louvre, in the presence of the genius of Poussin" (210) 

Poussin est présent partout dans le texte, y faisant sentir les multiples effets de ses réinterprétations personnelles et nécessairement subjectives, et cependant aussi toujours absent, nulle part "décrit». Pour reprendre le terme de Liliane Louvel, le degré de " saturation du texte par le pictural $»^{4}$ (Louvel 32) est ici à la fois maximal puisque l'image est omni-présente et que l'on voyage mentalement dans les scènes de l'Arcadie, et en même temps minimal, puisque le pictural ne se constitue jamais fermement en objet dans le texte. Et c'est peut-être bien là qu'est la différence fondamentale. Le texte de Okri ne propose jamais un discours sur un objet pictural, car le thème de Poussin ne parvient pas à s'y constituer en tableau. Il invente en quelque sorte ce que pourrait être le discours de l'œuvre ou le discours induit par l'œuvre. Au lieu de parler de l'œuvre, il fait donc parler l'œuvre, et c'est une distinction qui pourrait être à son tour opératoire dans les différentes manières de créer le rapport image-texte. Il y aurait en effet d'un côté les récits qui prennent le tableau pour sujet et qui tentent de l'achever, ou du moins de le 
sillonner, d'en faire le tour par le discours. Mais le danger est que le pictural y reste un objet passif, sinon mort, que le texte ne parvienne pas véritablement à activer. Je songe ici à tous ces romans contemporains qui ne considèrent guère le tableau que comme prétexte à raconter une histoire - sentimentale de préférence : La Jeune fille à la perle, ou La Dame à la licorne de Tracy Chevalier, ou encore Lydia Cassatt reading the morning papers, de Harriett Scott Chesman, titre qui trahit de manière symptomatique la focalisation sur le sujet peint (la sœur malade posant pour l'artiste), et non sur l'artiste elle-même, Mary Cassatt. D'un autre côté (et en réalité ce ne sont pas là deux catégories hermétiques mais les deux extrêmes d'un spectre qui déploie tous les degrés d'intégration active de l'image dans le texte), des textes où l'image ne fournit plus seulement le sujet (au sens passif de " subject matter ») mais devient une véritable dynamique du texte, quittant le domaine de la simple description ou même de l'image comme " embrayeur de narration ", pour devenir «moteur " ou agent de cette même narration. C'est ce que réussit à faire Ben Okri: exploiter le potentiel narratif du tableau, substituer au traitement purement thématique de l'image une exploitation dynamique. Ou pour reprendre les termes de Louis Marin, « non pas signifier ou expliquer le tableau, mais indiquer le difficile chemin vers la porte sacrée ouverte pour Enée par la Sibylle » (Marin 1977, 17).

\section{BIBLIOGRAPHIE}

Foucault, Michel. Histoire de la folie à l'âge classique. Paris : Gallimard, « coll. Tel », 1972.

Louvel, Liliane. Image - texte : images à lire, textes à voir. Rennes : PUR, 2002

Marin, Louis. Détruire la peinture. Paris : Champs Flammarion, 1977.

Marin, Louis. « Panofsky et Poussin en Arcadie ». Sublime Poussin. Paris : Seuil, 1995.

Mitchell, W.J.T. Picture Theory: Essays on Verbal and Visual Representation. U of Chicago P, 1994.

Okri, Ben. Arcadia (2002). London: Phoenix, 2003.

Panofsky, Erwin. « Et in Arcadia Ego : Poussin et la tradition élégiaque ». L'CEuvre d'art et ses

significations. Paris : Gallimard, 1969.

\section{NOTES}

1. Encylopédie Universalis, article « Arcadie », vol. 18, p. 95d.

2. Toutes les paginations renvoient à l'édition de 2003 (London: Phoenix, 2003 ; la première édition fut publiée en 2002 chez Weidenfeld and Nicholson).

3. Michel Foucault, Histoire de la folie à l'âge classique, Paris : Gallimard, 1972, « coll. Tel », 13-55.

4. L. Louvel parle de « saturation picturale du texte». 
INDEX

oeuvrecitee In Arcadia 